\title{
Sharp Efficiency for Vector Equilibrium Problems on Banach Spaces
}

\author{
Si-Huan Li, ${ }^{1,2}$ Qiang Wang, ${ }^{3}$ Shu Xu, ${ }^{4}$ and Jun-Xiang Wang ${ }^{5}$ \\ ${ }^{1}$ School of Economics and Business Administration, Chongqing University, Chongqing 400030, China \\ ${ }^{2}$ Department of Business Administration, Huaihua University, Huaihua, Hunan 418000, China \\ ${ }^{3}$ Automobile and Traffic Engineering College, Heilongjiang Institute of Technology, Harbin 150050, China \\ ${ }^{4}$ Rear Services Office, Chongqing Police College, Chongqing 401331, China \\ ${ }^{5}$ Office of Academic Affairs, Heilongjiang Institute of Technology, Harbin 150050, China
}

Correspondence should be addressed to Si-Huan Li; hhshsv008@163.com

Received 31 December 2012; Accepted 27 February 2013

Academic Editor: Qun Lin

Copyright (C) $2013 \mathrm{Si}$-Huan Li et al. This is an open access article distributed under the Creative Commons Attribution License, which permits unrestricted use, distribution, and reproduction in any medium, provided the original work is properly cited.

The concept of sharp efficient solution for vector equilibrium problems on Banach spaces is proposed. Moreover, the Fermat rules for local efficient solutions of vector equilibrium problems are extended to the sharp efficient solutions by means of the Clarke generalized differentiation and the normal cone. As applications, some necessary optimality conditions and sufficient optimality conditions for local sharp efficient solutions of a vector optimization problem with an abstract constraint and a vector variational inequality are obtained, respectively.

\section{Introduction}

Let $X, Y$ be Banach spaces, and let $0_{X}$ and $0_{Y}$ be the origins of $X$ and $Y$, respectively. Let $S$ be a nonempty subset of $X$ and $C \subset Y$ let be a closed convex pointed cone with apex at the origin. Let $Y$ be partially ordered by $C$ and let $F: X \times X \rightarrow Y$ be a bifunction. Consider the following vector equilibrium problem (for short, VEP):

$$
\text { find } \hat{x} \in S \text { such that } F(\widehat{x}, x) \notin-C \backslash\left\{0_{Y}\right\} \quad \forall x \in S \text {. }
$$

Recall from [1-4] that a vector $\hat{x} \in S$ is said to be a local efficient solution for VEP iff there exists a neighborhood $U$ of $\hat{x}$ such that

$$
F(\widehat{x}, x) \notin-C \backslash\left\{0_{Y}\right\}, \quad \forall x \in S \cap U,
$$

In particular, if $U=X$, then $\hat{x}$ is said to be an efficient solution for (VEP). In this paper, we pay our attention to the following stronger efficient solution, called local sharp efficient solution, for VEP.

Definition 1. A vector $\hat{x} \in S$ is said to be a local sharp efficient solution for (VEP) iff there exist a neighborhood $U$ of $\hat{x}$ and a real number $\eta>0$ such that

$$
F(\widehat{x}, x) \notin-C+\eta\|x-\widehat{x}\| B_{Y}, \quad \forall x \in S \cap U \backslash\{\hat{x}\},
$$

where $B_{Y}$ denotes the open unit ball of $Y$. particularly, if $U=$ $X$, then $\widehat{x}$ is said to be a sharp efficient solution for (VEP).

Remark 2. (i) Note that it always holds $F(\widehat{x}, \widehat{x})=0_{Y}$ for several major classes of special problems, such as VOP, and VVI. Based on this fact, we consider all the points around $\hat{x}$ in $S$ except $\widehat{x}$ in Definition 1 since $0_{Y} \in-C$.

(ii) If $\hat{x} \in S$ satisfying $F(\hat{x}, \widehat{x})=0_{Y}$ is a local sharp efficient solution for (VEP), then it is obvious that $\widetilde{S} \cap U=\{\widehat{x}\}$; that is, $\widehat{x}$ is an isolated point of $\widetilde{S}$.

(iii) Every local sharp efficient solution must be a local efficient solution for (VEP).

As we know, vector equilibrium problems cover various classes of optimization-related problems and models arisen in practical applications, such as vector variational inequalities, vector optimization problems, vector Nash equilibrium problems, and vector complementarity problem, see [5-10] and references therein. Particularly, if we take 
$F(\widehat{x}, x)=f(x)-f(\widehat{x})$ for all $x, \widehat{x} \in X$ with $f: X \rightarrow Y$ being a vector-valued map, then (VEP) reduces to the following vector optimization problem (for short, VOP):

$$
\min f(x), \quad \text { s.t. } x \in S \text {. }
$$

In the sequel, a vector $\hat{x} \in S$ is said to be a local sharp efficient solution (resp., sharp efficient solution) for VOP if and only if it is a local sharp efficient solution (resp., sharp efficient solution) for VEP; that is, there exist a neighborhood $U$ (resp., $U=X)$ of $\hat{x}$ and a real number $\eta>0$ such that

$$
\begin{array}{r}
(f(x)+C) \cap \mathscr{B}(f(\hat{x}), \eta\|x-\hat{x}\|)=\varnothing, \\
\forall x \in S \cap U \backslash\{\hat{x}\},
\end{array}
$$

where $\mathscr{B}(f(\hat{x}), \eta\|x-\hat{x}\|)$ denotes the open ball with center at $f(\widehat{x})$ and radius $\eta\|x-\widehat{x}\|$. It is well known that the previous definition of local sharp efficient solution (resp., sharp efficient solution) for (VOP) is first proposed by Jiménez in [11], which is a natural generalization of the isolated local minima for scalar optimization problems. For more details, we refer to [12-17] and references therein. Analogously, if we take $F(\hat{x}, x)=\langle T(\widehat{x}), x-\widehat{x}\rangle$, where $T: X \rightarrow \mathbb{L}(X, Y)$ is a mapping and $\llbracket(X, Y)$ denotes the set of all linear continuous operators from $X$ to $Y$, then (VEP) reduces to the vector variational inequalities (for short, VVI) as follows:

find $\hat{x} \in S$ such that $\langle T(\hat{x}), x-\widehat{x}\rangle \notin-C \backslash\left\{0_{Y}\right\} \quad \forall x \in S$.

Moreover, a vector $\widehat{x} \in S$ is said to be a local sharp efficient solution (resp., sharp efficient solution) for VVI if and only if it is local sharp efficient solution (resp., sharp efficient solution) for VEP; that is, there exist a neighborhood $U$ (resp., $U=X)$ of $\widehat{x}$ and a real number $\eta>0$ such that

$$
\langle T(\hat{x}), x-\hat{x}\rangle \notin-C+\eta\|x-\widehat{x}\| B_{Y}, \quad \forall x \in S \cap U \backslash\{\hat{x}\} .
$$

Recently, there has been increasing interest in dealing with optimality conditions for nonsmooth optimization problems by virtue of modern variational analysis techniques. Gong [4] established some necessary conditions for weakly efficient solutions, Henig efficient solutions, globally efficient solutions, and superefficient solutions to vector equilibrium problems by using nonsmooth analysis. By means of convex analysis and nonsmooth analysis, Yang and Zheng [18] provided some sufficient conditions and necessary conditions for a point to be an approximate solution of vector variational inequalities. In [15], Zheng et al. studied sharp minima for multiobjective optimization problems in terms of the Mordukhovich coderivative and the normal cone and presented some optimality conditions. Moreover, Zhu et al. [19] extended the Fermat rules for the local minima of the constrained set-valued optimization problem to the sharp minima and the weak sharp minima in Banach spaces or Asplund spaces, by means of the Mordukhovich generalized differentiation and the normal cone.

In this paper, by virtue of the Clarke generalized differentiation and the normal cone, we first establish a necessary optimality condition for the local sharp efficient solution of (VEP) without any convexity assumptions. And then, we obtain the sufficient optimality condition for the local sharp efficient solution of (VEP) under some appropriate convexity assumptions. Simultaneously, we show that the local sharp efficient solution and the sharp efficient solution are equivalent for the convex case. Finally, we apply our results, respectively, to get some necessary optimality conditions and sufficient optimality conditions for local sharp efficient solutions of a vector optimization problem with an abstract constraint and a vector variational inequality.

\section{Notations and Preliminaries}

Throughout this paper, we denote by int $S$ the interior of $S$ and $w^{*}$ the weak star topology on dual spaces. As usual, the distance function $d(\bullet, S): X \rightarrow \mathbb{R}$, the indicator function $\psi(\bullet, S): X \rightarrow \mathbb{R} \cup\{+\infty\}$, and the support function $\psi^{*}(\bullet, S):$ $X^{*} \rightarrow \mathbb{R} \cup\{+\infty\}$ for $S$ are, respectively, defined by

$$
\begin{gathered}
d(x, S):=\inf _{y \in S}\|x-y\|, \quad \forall x \in X, \\
\psi(x, S):=0 \quad \text { if } x \in S, \quad \psi(x, S):=+\infty, \quad \text { if } x \notin S, \quad(8) \\
\psi^{*}\left(x^{*}, S\right):=\sup _{x \in S}\left\langle x^{*}, x\right\rangle, \quad \forall x^{*} \in X^{*} .
\end{gathered}
$$

The main tools for our study in the paper are the Clarke generalized differentiation notions which are generally used in variational analysis and nonsmooth analysis. We refer to $[13,20-23]$ and references therein for more details. Recall that the vector-valued map $F: X \rightarrow Y$ is said to be Fréchet differentiable at $\widehat{x}$ if there exists a linear continuous operator $\nabla F(\widehat{x}): X \rightarrow Y$ such that

$$
\lim _{x \rightarrow \widehat{x}} \frac{F(x)-F(\widehat{x})-\nabla F(\widehat{x})(x-\widehat{x})}{\|x-\hat{x}\|}=0_{Y}
$$

Here, $\nabla F(\hat{x})$ is called the Fréchet derivative of $F$ at $\widehat{x}$. As usual, we denote by $(\nabla F(\hat{x}))^{*}: Y^{*} \rightarrow X^{*}$ the adjoint operator of $\nabla F(\widehat{x})$; that is, $\langle\nabla F(\widehat{x})(x), y\rangle=\left\langle x,(\nabla F(\widehat{x}))^{*}(y)\right\rangle$ for all $x \in X$ and $y \in Y$. Moreover, $F$ is said to be strictly differentiable at $\widehat{x}$ if

$$
\lim _{x \rightarrow \widehat{x}, u \rightarrow \widehat{x}, x \neq u} \frac{F(x)-F(u)-\nabla F(\hat{x})(x-u)}{\|x-u\|}=0_{Y} .
$$

Let $h: X \rightarrow \mathbb{R} \cup\{+\infty\}$ be a proper lower semicontinuous function. Recall that the Clarke-Rockafellar generalized directional derivative $h^{\uparrow}(\widehat{x}, d)$ of $h$ at $\widehat{x} \in \operatorname{dom} h:=\{x \in X \mid$ $h(x)<+\infty\}$ in the direction $d \in X$ is defined by

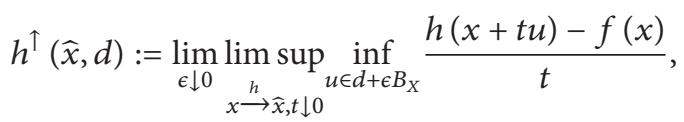

where $x \stackrel{h}{\rightarrow} \hat{x}$ means $x \rightarrow \widehat{x}$ and $h(x) \rightarrow h(\widehat{x})$. The Clarke subdifferential $\partial_{c} h(\hat{x})$ of $h$ at $\hat{x} \in \operatorname{dom} h$ is defined as

$$
\partial_{c} h(\widehat{x}):=\left\{x^{*} \in X^{*} \mid\left\langle x^{*}, d\right\rangle \leq h^{\uparrow}(\widehat{x}, d), \forall d \in X\right\} .
$$


Furthermore, if $h$ is a convex function, then $\partial_{c} h(\hat{x})$ is in accordance with the subdifferential $\partial h(\widehat{x})$ in convex analysis; that is,

$$
\begin{aligned}
& \partial_{c} h(\widehat{x}) \\
& =\partial h(\widehat{x}) \\
& \quad=\left\{x^{*} \in \mathbb{R}^{n} \mid\left\langle x^{*}, x-\widehat{x}\right\rangle \leq h(x)-h(\widehat{x}), \forall x \in X\right\} .
\end{aligned}
$$

Given a point $\hat{x} \in S$. Recall that the Clarke tangent cone $T_{c}(S, \hat{x})$ of $S$ at $\hat{x}$ is

$$
\begin{aligned}
& T_{c}(S, \widehat{x}) \\
& \quad:=\left\{v \in X \mid \forall x_{n} \longrightarrow \widehat{x}, \forall t_{n} \longrightarrow 0^{+},\right. \\
& \left.\quad \exists v_{n} \longrightarrow v \text { s.t. } x_{n}+t_{n} v_{n} \in S, \forall n \in \mathbb{N}\right\} .
\end{aligned}
$$

The Clarke normal cone $N_{c}(S, \hat{x})$ of $S$ at $\hat{x}$ is defined by the polar of $T_{c}(S, \widehat{x})$; that is,

$$
\begin{aligned}
& N_{c}(S, \widehat{x}) \\
& \quad:=T_{c}(S, \widehat{x})^{\circ} \\
& :=\left\{x^{*} \in X^{*} \mid\left\langle x^{*}, v\right\rangle \leq 0, \forall v \in T_{c}(S, \widehat{x})\right\} .
\end{aligned}
$$

In particular, if $S$ is convex, then $N_{c}(S, \hat{x})$ agrees with the normal cone $N(S, \hat{x})$ of convex analysis; that is,

$$
\begin{aligned}
& N_{c}(S, \widehat{x}) \\
& \quad=N(S, \widehat{x}) \\
& \quad=\left\{x^{*} \in X^{*} \mid\left\langle x^{*}, x-\widehat{x}\right\rangle \leq 0, \forall x \in S\right\},
\end{aligned}
$$

and if $S$ is closed, then we have $\partial_{c} \psi(\hat{x}, S)=N_{c}(S, \widehat{x})$ for all $x \in S$.

Next, we collect some useful and important propositions for this paper.

Proposition 3. For every nonempty closed subset $\Omega \subset X$ and every $x \in \Omega$, one has $\partial_{c} d(\bullet, \Omega)(x) \subset \mathbb{B}_{X^{*}} \cap N_{c}(\Omega, x)$ and $N_{c}(\Omega, x)=\mathrm{cl}^{*} \quad\left\{\bigcup_{\lambda>0} \lambda \partial_{c} d(\bullet, \Omega)(x)\right\}$, where $\mathbb{B}_{X^{*}}$ and $\mathrm{cl}^{*}$ denote the closed unit ball of $X^{*}$ and the $w^{*}$-closure, respectively.

The following necessary optimality condition, called generalized Fermat rule, for a function to attain its local minimum is useful for our analysis.

Proposition 4 (generalized Fermat rule). Let $f: X \rightarrow \mathbb{R} \cup$ $\{+\infty\}$ be a proper lower semicontinuous function. If $f$ attains a local minimum at $\widehat{x} \in X$, then $0_{X^{*}} \in \partial_{c} f(\hat{x})$.

We recall the following sum rule for the Clarke subdifferential which is important in the sequel.

Proposition 5. Let $f, h: X \rightarrow \mathbb{R} \cup\{+\infty\}$ be proper lower semicontinuous functions and $x \in \operatorname{dom} f \cap \operatorname{dom} h$. If $f$ is locally Lipschitz around $x$, then $\partial_{c}(f+h)(x) \subset \partial_{c} f(x)+\partial_{c} h(x)$.
The following chain rule of Clarke subdifferential is useful in the paper.

Proposition 6. Let $X, Y$ be Banach spaces, Let $G: X \rightarrow Y$ be a vector-valued map, and Let $g: Y \rightarrow \mathbb{R} \cup\{+\infty\}$ be a real-valued function. Suppose that $G$ is strictly differentiable at $x$ and $g$ is locally Lipschitz around $G(x)$. Then $f=g \circ G$ : $X \rightarrow \mathbb{R} \cup\{+\infty\}$ is locally Lipschitz around $x$, and one has

$$
\partial_{c} f(x) \subset\left(G^{\prime}(x)\right)^{*} \partial_{c} g(G(x)) .
$$

\section{Optimality Conditions}

Given a point $\hat{x} \in S$. In the sequel, let the vector-valued map $F_{\widehat{x}}: X \rightarrow Y$ be defined by

$$
F_{\widehat{x}}(x):=F(\widehat{x}, x), \quad \forall x \in X .
$$

Theorem 7 (strong Fermat rule). Given a point $\hat{x} \in S$ with $F(\widehat{x}, \widehat{x})=0_{Y}$. Suppose that $S$ is a closed subset of $X$.

(i) If $\hat{x}$ is a local sharp efficient solution for (VEP) and $F_{\widehat{x}}$ is strictly differentiable at $\hat{x}$, then one has

$$
0_{X^{*}} \in \operatorname{int}\left(\left(\nabla F_{\widehat{x}}(\widehat{x})\right)^{*}\left(C^{+} \cap \mathbb{B}_{Y^{*}}\right)+N_{c}(S, \widehat{x})\right) .
$$

(ii) Let $S$ be convex and let $F_{\widehat{x}}$ be $C$-convex on $S$. Assume that $F_{\widehat{x}}$ is Fréchet differentiable at $\widehat{x}$. Then it follows that

$$
0_{X^{*}} \in \operatorname{int}\left(\left(\nabla F_{\widehat{x}}(\widehat{x})\right)^{*}\left(C^{+} \cap \mathbb{B}_{Y^{*}}\right)+N(S, \widehat{x})\right)
$$

$$
\text { implies } \widehat{x} \text { being a sharp efficient solution for (VEP). }
$$

Proof. (i) Since $\hat{x} \in S$ is a local sharp efficient solution for (VEP), there exist a neighborhood $U$ of $\widehat{x}$ and a real number $\eta>0$ such that

$$
F(\widehat{x}, x) \notin-C+\eta\|x-\widehat{x}\| B_{Y}, \quad \forall x \in S \cap U \backslash\{\widehat{x}\},
$$

which implies that

$$
d\left(F_{\widehat{x}}(x),-C\right)+\psi(x, S) \geq \eta\|x-\hat{x}\|, \quad \forall x \in U .
$$

Take arbitrary $x^{*} \in \mathbb{B}_{X^{*}}$. Then we have $\left\langle x^{*}, x-\hat{x}\right\rangle \leq\|x-\hat{x}\|$ for all $x \in X$. Together with (22), it follows that $\hat{x} \in S$ is a local minimum point of the function $f: X \rightarrow \mathbb{R}$ defined by

$$
\begin{array}{r}
f(x):=-\eta\left\langle x^{*}, x-\widehat{x}\right\rangle+d\left(F_{\widehat{x}}(x),-C\right)+\psi(x, S), \\
\forall x \in X .
\end{array}
$$

By Proposition 4 (generalized Fermat rule), we have $0_{X^{*}} \epsilon$ $\partial f(\widehat{x})$. Note that $F_{\widehat{x}}$ is strictly differentiable at $\hat{x}$ and the distance function is globally Lipschitz with modulus 1 . It follows from Proposition 6 that $d\left(F_{\widehat{x}}(\bullet),-C\right): X \rightarrow \mathbb{R}$ is locally Lipschitz around $\hat{x}$, and, moreover, together with Proposition $3, F(\hat{x}, \widehat{x})=0_{Y}$ and $N_{c}\left(-C, 0_{Y}\right)=C^{+}$, one has

$$
\partial_{c} d\left(F_{\widehat{x}}(\bullet),-C\right)(\widehat{x}) \subset\left(\nabla F_{\widehat{x}}(\widehat{x})\right)^{*}\left(C^{+} \cap \mathbb{B}_{Y^{*}}\right) .
$$


Furthermore, $S$ is closed, which implies that $\psi$ is lower semicontinuous function. Thus, by Proposition 5 and (24), we have

$$
\eta x^{*} \in\left(\nabla F_{\widehat{x}}(\widehat{x})\right)^{*}\left(C^{+} \cap \mathbb{B}_{Y^{*}}\right)+N_{c}(S, \widehat{x}) .
$$

Since $x^{*} \in \mathbb{B}_{X^{*}}$ is arbitrary, it follows that $\eta \mathbb{B}_{X^{*}} \subset$ $\left(\nabla F_{\widehat{x}}(\hat{x})\right)^{*}\left(C^{+} \cap \mathbb{B}_{Y^{*}}\right)+N_{c}(S, \widehat{x})$, which implies that

$$
0_{X^{*}} \in \operatorname{int}\left(\left(\nabla F_{\widehat{x}}(\widehat{x})\right)^{*}\left(C^{+} \cap \mathbb{B}_{Y^{*}}\right)+N_{c}(S, \widehat{x})\right) .
$$

(ii) Since $0_{X^{*}} \in \operatorname{int}\left(\left(\nabla F_{\widehat{x}}(\widehat{x})\right)^{*}\left(C^{+} \cap \mathbb{B}_{Y^{*}}\right)+N(S, \widehat{x})\right)$, there exists some real number $\eta>0$ such that

$$
\eta \mathbb{B}_{X^{*}} \subset\left(\nabla F_{\widehat{x}}(\widehat{x})\right)^{*}\left(C^{+} \cap \mathbb{B}_{Y^{*}}\right)+N(S, \widehat{x}) .
$$

Take arbitrary $x^{*} \in \mathbb{B}_{X^{*}}$. Then there exist $y^{*} \in C^{+} \cap \mathbb{B}_{Y^{*}}$ and $z^{*} \in N(S, \widehat{x})$ such that

$$
\eta x^{*}=\left(\nabla F_{\widehat{x}}(\widehat{x})\right)^{*}\left(y^{*}\right)+z^{*} .
$$

Since $F_{\widehat{x}}$ is $C$-convex on $S$, we have ([24, Theorem 2.20])

$$
F_{\widehat{x}}(\widehat{x})+\nabla F_{\widehat{x}}(\widehat{x})(x-\widehat{x}) \in F_{\widehat{x}}(x)-C, \quad \forall x \in S .
$$

Together with $y^{*} \in C^{+} \cap \mathbb{B}_{Y^{*}}$, we have

$$
\begin{aligned}
& \left\langle\left(\nabla F_{\widehat{x}}(\widehat{x})\right)^{*}\left(y^{*}\right), x-\widehat{x}\right\rangle \\
& \quad=\left\langle y^{*}, \nabla F_{\widehat{x}}(\widehat{x})(x-\widehat{x})\right\rangle \\
& \quad \leq\left\langle y^{*}, F_{\widehat{x}}(x)\right\rangle \\
& \quad \leq\left\langle y^{*}, F_{\widehat{x}}(x)+c\right\rangle \\
& \quad \leq\left\|F_{\widehat{x}}(x)+c\right\|, \quad \forall c \in C, \forall x \in S,
\end{aligned}
$$

which implies that

$$
\left\langle\left(\nabla F_{\widehat{x}}(\widehat{x})\right)^{*}\left(y^{*}\right), x-\widehat{x}\right\rangle \leq d\left(F_{\widehat{x}}(x),-C\right), \quad \forall x \in S .
$$

Moreover, it follows from $S$ being convex and $z^{*} \in N(S, \widehat{x})$ that $\left\langle z^{*}, x-\widehat{x}\right\rangle \leq 0$ for all $x \in S$. Together with (28) and (31), we can conclude that

$$
\left\langle\eta x^{*}, x-\hat{x}\right\rangle \leq d\left(F_{\widehat{x}}(x),-C\right), \quad \forall x \in S
$$

Since $x^{*} \in \mathbb{B}_{X^{*}}$ is arbitrary, we have

$$
\begin{aligned}
\eta\|x-\hat{x}\| & =\sup _{x^{*} \in \mathbb{B}_{X^{*}}}\left\langle\eta x^{*}, x-\widehat{x}\right\rangle \\
& \leq d\left(F_{\widehat{x}}(x),-C\right), \quad \forall x \in S,
\end{aligned}
$$

that is,

$$
F(\widehat{x}, x) \notin-C+\eta\|x-\hat{x}\| B_{Y}, \quad \forall x \in S \backslash\{\widehat{x}\} .
$$

Thus, $\hat{x}$ is a sharp efficient solution for (VEP).
Remark 8. Assume that $S$ is convex and $F_{\widehat{x}}$ is Fréchet differentiable at $\hat{x}$. Then it is obvious that

$$
0_{X^{*}} \in \operatorname{int}\left(\left(\nabla F_{\widehat{x}}(\widehat{x})\right)^{*}\left(\mathrm{C}^{+} \cap \mathbb{B}_{Y^{*}}\right)+N(S, \widehat{x})\right)
$$

Implies that

$$
0_{X^{*}} \in \operatorname{int}\left(\left(\nabla F_{\widehat{x}}(\widehat{x})\right)^{*}\left(C^{+}\right)+N(S, \widehat{x})\right) .
$$

Since $\left(\nabla F_{\widehat{x}}(\widehat{x})\right)^{*}\left(C^{+}\right)+N(S, \widehat{x})$ is a cone, we have

$$
\left(\nabla F_{\widehat{x}}(\widehat{x})\right)^{*}\left(C^{+}\right)+N(S, \widehat{x})=X^{*} .
$$

It is worth noting that (37) implies (35); that is, (35), (36), and (37) are equivalent. In the following lemma, we prove this assertion.

Lemma 9. Let $S$ be convex and let $F_{\widehat{x}}$ be Fréchet differentiable at $\widehat{x}$. Then (35), (36) and (37) are equivalent.

Proof. We only need to prove that (37) implies (35). It follows from (37) that

$$
X^{*}=\bigcup_{n=1}^{+\infty} \mathrm{cl}\left(\left(\nabla F_{\widehat{x}}(\widehat{x})\right)^{*}\left(n \mathbb{B}_{Y^{*}} \cap C^{+}\right)+n \mathbb{B}_{X^{*}} \cap N(S, \widehat{x})\right) .
$$

Note that the dual space $X^{*}$ is complete. By (38) and the Baire's category theorem ([25, Theorem 4.7-2]) there exists some $n_{0} \in \mathbb{N}$ such that

$$
\text { int } \operatorname{cl}\left(\left(\nabla F_{\widehat{x}}(\widehat{x})\right)^{*}\left(n_{0} \mathbb{B}_{Y^{*}} \cap C^{+}\right)+n_{0} \mathbb{B}_{X^{*}} \cap N(S, \widehat{x})\right) \neq \varnothing \text {. }
$$

Thus, we have int $\operatorname{cl}\left(\left(\nabla F_{\widehat{x}}(\widehat{x})\right)^{*}\left(\mathbb{B}_{Y^{*}} \cap C^{+}\right)+\mathbb{B}_{X^{*}} \cap N(S, \hat{x})\right)$ $\neq \varnothing$. Moreover, we can conclude that

$$
\operatorname{int}\left(\left(\nabla F_{\widehat{x}}(\widehat{x})\right)^{*}\left(\mathbb{B}_{Y^{*}} \cap C^{+}\right)+\mathbb{B}_{X^{*}} \cap N(S, \widehat{x})\right) \neq \varnothing
$$

since $\left(\nabla F_{\widehat{x}}(\hat{x})\right)^{*}\left(\mathbb{B}_{Y^{*}} \cap C^{+}\right)+\mathbb{B}_{X^{*}} \cap N(S, \widehat{x})$ is closed. In fact, take arbitrary sequence $\left\{x_{n}^{*}\right\} \subset\left(\nabla F_{\widehat{x}}(\widehat{x})\right)^{*}\left(\mathbb{B}_{Y^{*}} \cap C^{+}\right)+$ $\mathbb{B}_{X^{*}} \cap N(S, \hat{x})$ converging to some $x^{*} \in X^{*}$. Then, there exist $u_{n}^{*} \in \mathbb{B}_{Y^{*}} \cap C^{+}$and $v_{n}^{*} \in \mathbb{B}_{X^{*}} \cap N(S, \hat{x})$ such that $x_{n}^{*}=\left(\nabla F_{\widehat{x}}(\widehat{x})\right)^{*}\left(u_{n}^{*}\right)+v_{n}^{*}$ for all $n \in \mathbb{N}$. Since the closed unit ball of the dual space of a Banach space is weak ${ }^{*}$-compact, without loss of generality, we can assume that

$$
u_{n}^{*} \stackrel{w^{*}}{\longrightarrow} u^{*} \in \mathbb{B}_{Y^{*}} \cap C^{+}, \quad v_{n}^{*} \stackrel{w^{*}}{\longrightarrow} v^{*} \in \mathbb{B}_{X^{*}}
$$

(passing to a subsequence if necessary). Moreover, since the conjugate operator $\left(\nabla F_{\widehat{x}}(\widehat{x})\right)^{*}$ is weak ${ }^{*}$-weak ${ }^{*}$ continuous, we get

$$
x^{*}=\left(\nabla F_{\widehat{x}}(\widehat{x})\right)^{*}\left(u^{*}\right)+v^{*} .
$$

Since $S$ is convex, it follows from $v_{n}^{*} \in \mathbb{B}_{X^{*}} \cap N(S, \widehat{x})$ that

$$
\left\langle v_{n}^{*}, x-\widehat{x}\right\rangle \leq 0, \quad \forall n \in \mathbb{N}, \forall x \in S .
$$

Thus, we have $\left\langle v^{*}, x-\hat{x}\right\rangle \leq 0, \forall x \in S$; that is, $v^{*} \in N(S, \hat{x})$. Together with (41) and (42), it follows that $x^{*} \in\left(\nabla F_{\widehat{x}}(\widehat{x})\right)^{*}\left(\mathbb{B}_{Y^{*}} \cap C^{+}\right)+\mathbb{B}_{X^{*}} \cap N(S, \widehat{x})$. Therefore, 
we have shown that $\left(\nabla F_{\widehat{x}}(\widehat{x})\right)^{*}\left(\mathbb{B}_{Y^{*}} \cap C^{+}\right)+\mathbb{B}_{X^{*}} \cap N(S, \widehat{x})$ is closed. By (40), there exist some $x_{0}^{*} \in X^{*}$ and $\rho>0$ such that

$$
x_{0}^{*}+\rho \mathbb{B}_{X^{*}} \subset\left(\nabla F_{\widehat{x}}(\widehat{x})\right)^{*}\left(\mathbb{B}_{Y^{*}} \cap C^{+}\right)+\mathbb{B}_{X^{*}} \cap N(S, \widehat{x}) .
$$

Moreover, since $-x_{0}^{*} \in X^{*}$, it follows from (38) that there exists some $n_{1} \in \mathbb{N}$ such that $-x_{0}^{*} \in \mathrm{cl}\left(\left(\nabla F_{\widehat{X}}(\widehat{x})\right)^{*}\left(n_{1} \mathbb{B}_{Y^{*}} \cap\right.\right.$ $\left.\left.C^{+}\right)+n_{1} \mathbb{B}_{X^{*}} \cap N(S, \widehat{x})\right)$. Together with the closedness of $\left(\nabla F_{\widehat{x}}(\widehat{x})\right)^{*}\left(\mathbb{B}_{Y^{*}} \cap C^{+}\right)+\mathbb{B}_{X^{*}} \cap N(S, \widehat{x})$, we have

$$
-\frac{1}{n_{1}} x_{0}^{*} \in\left(\nabla F_{\widehat{x}}(\widehat{x})\right)^{*}\left(\mathbb{B}_{Y^{*}} \cap C^{+}\right)+\mathbb{B}_{X^{*}} \cap N(S, \widehat{x}) .
$$

Since $C^{+}$and $N(S, \widehat{x})$ are convex cones, we get from (44) that

$$
\begin{aligned}
\frac{\rho}{n_{1}} \mathbb{B}_{X^{*}} \subset & \left(1+\frac{1}{n_{1}}\right) \\
& \times\left(\left(\nabla F_{\widehat{x}}(\widehat{x})\right)^{*}\left(\mathbb{B}_{Y^{*}} \cap C^{+}\right)+\mathbb{B}_{X^{*}} \cap N(S, \widehat{x})\right),
\end{aligned}
$$

that is,

$$
\frac{\rho}{n_{1}+1} \mathbb{B}_{X^{*}} \subset\left(\nabla F_{\widehat{x}}(\widehat{x})\right)^{*}\left(\mathbb{B}_{Y^{*}} \cap C^{+}\right)+\mathbb{B}_{X^{*}} \cap N(S, \widehat{x}) .
$$

Thus, $0_{X^{*}} \in \operatorname{int}\left(\left(\nabla F_{\widehat{x}}(\widehat{x})\right)^{*}\left(\mathbb{B}_{Y^{*}} \cap C^{+}\right)+\mathbb{B}_{X^{*}} \cap N(S, \widehat{x})\right)$, which implies that

$$
0_{X^{*}} \in \operatorname{int}\left(\left(\nabla F_{\widehat{x}}(\widehat{x})\right)^{*}\left(C^{+} \cap \mathbb{B}_{Y^{*}}\right)+N(S, \widehat{x})\right) .
$$

This completes the proof.

Remark 10. In the proof of Theorem 7(i), we have shown that if $F_{\widehat{x}}$ is strictly differentiable at $\hat{x}$ and $F(\hat{x}, \widehat{x})=0_{Y}$, then $\partial_{c} d\left(F_{\widehat{x}}(\bullet),-C\right)(\widehat{x}) \subset\left(\nabla F_{\widehat{x}}(\widehat{x})\right)^{*}\left(C^{+} \cap \mathbb{B}_{Y^{*}}\right)$. Moreover, if, in addition, $F_{\widehat{x}}$ is $C$-convex on $X$, then we have

$$
\partial_{c} d\left(F_{\widehat{x}}(\bullet),-C\right)(\widehat{x})=\left(\nabla F_{\widehat{x}}(\widehat{x})\right)^{*}\left(C^{+} \cap \mathbb{B}_{Y^{*}}\right) .
$$

We give the following lemma to explain this result.

Lemma 11. Let $F_{\widehat{x}}$ be strictly differentiable at $\widehat{x}$ and $F(\widehat{x}, \widehat{x})=$ $0_{Y}$. Suppose that $F_{\widehat{x}}$ is $C$-convex on $X$; then it follows that

$$
\partial d\left(F_{\widehat{x}}(\bullet),-C\right)(\widehat{x})=\left(\nabla F_{\widehat{x}}(\widehat{x})\right)^{*}\left(C^{+} \cap \mathbb{B}_{Y^{*}}\right) .
$$

Proof. Since $C$ is a convex cone, the real-valued function $d(\bullet,-C): Y \rightarrow \mathbb{R}$ is monotonically increasing; that is, $\forall y_{1}, y_{2} \in Y, y_{1} \leq_{C} y_{2}$ implies that $d\left(y_{1},-C\right) \leq d\left(y_{2},-C\right)$. Together with $F_{\hat{x}}$ is $C$-convex, we have that ([24, Lemma $2.7(\mathrm{~b})]) d\left(F_{\widehat{x}}(\bullet),-C\right): X \rightarrow \mathbb{R}$ being convex. By the proof of Theorem $7(\mathrm{i})$, it is sufficient to prove that $\left(\nabla F_{\widehat{x}}(\hat{x})\right)^{*}\left(C^{+} \cap\right.$ $\left.\mathbb{B}_{Y^{*}}\right) \subset \partial d\left(F_{\widehat{x}}(\bullet),-C\right)(\widehat{x})$. Since $C$ is a closed and convex cone, and $(-C)^{\circ}=C^{+}$, it follows that $([26$, Theorem 3.1$])$

$$
\begin{aligned}
d\left(F_{\widehat{x}}(x),-C\right) & =\psi^{*}\left(F_{\widehat{x}}(x), C^{+} \cap \mathbb{B}_{Y^{*}}\right) \\
& =\sup _{u^{*} \in C^{+} \cap \mathbb{B}_{Y^{*}}}\left\langle u^{*}, F_{\widehat{x}}(x)\right\rangle, \quad \forall x \in X .
\end{aligned}
$$

Note that $F_{\widehat{x}}$ is $C$-convex on $X$ and Fréchet differentiable at $\widehat{x}$, and $F(\widehat{x}, \widehat{x})=0_{Y}$. Then we get $\left(\left[24\right.\right.$, Theorem 2.20]) $\nabla F_{\widehat{x}}(x-$ $\widehat{x}) \in F_{\widehat{x}}(x)-C$ for all $x \in X$. Thus, we have

$$
\begin{aligned}
& \sup _{u^{*} \in C^{+} \cap \mathbb{B}_{Y^{*}}}\left\langle u^{*}, \nabla F_{\widehat{x}}(\widehat{x})(x-\widehat{x})\right\rangle \\
& \quad \leq \sup _{u^{*} \in C^{+} \cap \mathbb{B}_{Y^{*}}}\left\langle u^{*}, F_{\widehat{x}}(x)\right\rangle \\
& \quad=d\left(F_{\widehat{x}}(x),-C\right)-d\left(F_{\widehat{x}}(\widehat{x}),-C\right), \quad \forall x \in X .
\end{aligned}
$$

Then, for every $u^{*} \in C^{+} \cap \mathbb{B}_{Y^{*}}$, it follows that $\left\langle u^{*}, \nabla F_{\widehat{x}}(\widehat{x})(x-\widehat{x})\right\rangle=\left\langle\left(\nabla F_{\widehat{x}}(\widehat{x})\right)^{*} u^{*}, x-\widehat{x}\right\rangle \leq d\left(F_{\widehat{x}}(x),-C\right)-$ $d\left(F_{\hat{x}}(\hat{x}),-C\right), \forall x \in X$; that is, $u^{*} \in \partial d\left(F_{\widehat{x}}(\bullet),-C\right)(\hat{x})$. Since $u^{*} \in C^{+} \cap \mathbb{B}_{Y^{*}}$ is arbitrary, we can conclude that $\left(\nabla F_{\widehat{x}}(\widehat{x})\right)^{*}\left(C^{+} \cap \mathbb{B}_{Y^{*}}\right) \subset \partial d\left(F_{\widehat{x}}(\bullet),-C\right)(\widehat{x})$.

By Theorem 7 and Lemmas 9 and 11, we immediately have the the following characterization of the sharp efficiency for (VEP) in convex case.

Corollary 12. Given a point $\hat{x} \in S$ with $F(\hat{x}, \hat{x})=0_{Y}$. Let $S$ be a closed and convex subset of $X$, and let $F_{\hat{x}}$ be $C$-convex on $S$. Suppose that $F_{\hat{x}}$ is strictly differentiable at $\hat{x}$. Then the following assertions are equivalent:

(i) $\hat{x}$ is a local sharp efficient solution for (VEP),

(ii) $\hat{x}$ is a sharp efficient solution for (VEP),

(iii) $0_{X^{*}} \in \operatorname{int}\left(\partial d\left(F_{\widehat{x}}(\bullet),-C\right)(\widehat{x})+N(S, \widehat{x})\right)$,

(iv) $0_{X^{*}} \in \operatorname{int}\left(\left(\nabla F_{\widehat{x}}(\widehat{x})\right)^{*}\left(C^{+} \cap \mathbb{B}_{Y^{*}}\right)+N(S, \widehat{x})\right)$,

(v) $0_{X^{*}} \in \operatorname{int}\left(\left(\nabla F_{\widehat{x}}(\widehat{x})\right)^{*}\left(C^{+}\right)+N(S, \widehat{x})\right)$,

(vi) $\left(\nabla F_{\widehat{x}}(\widehat{x})\right)^{*}\left(C^{+}\right)+N(S, \widehat{x})=X^{*}$.

\section{Applications}

We devote this section to appling the obtained results in Section 3 to vector optimization problems and vector variational inequalities, respectively.

Theorem 13. Let $\hat{x} \in S$ and let $f: X \rightarrow Y$ be a mapping. Suppose that $S$ is a closed subset of $X$.

(i) If $\hat{x}$ is a local sharp efficient solution for (VOP) and $f$ is strictly differentiable at $\hat{x}$, then one has

$$
0_{X^{*}} \in \operatorname{int}\left((\nabla F(\widehat{x}))^{*}\left(C^{+} \cap \mathbb{B}_{Y^{*}}\right)+N_{c}(S, \widehat{x})\right) .
$$

(ii) Suppose that $S$ is convex, $f$ is strictly differentiable at $\hat{x}$ and $C$-convex on $S$. Then the following assertions are equivalent:

(a) $\hat{x}$ is local sharp efficient solution for (VOP),

(b) $\hat{x}$ is sharp efficient solution for (VOP),

(c) $0_{X^{*}} \in \operatorname{int}\left((\nabla F(\widehat{x}))^{*}\left(C^{+} \cap \mathbb{B}_{Y^{*}}\right)+N(S, \widehat{x})\right)$,

(d) $0_{X^{*}} \in \operatorname{int}\left((\nabla F(\widehat{x}))^{*}\left(C^{+}\right)+N(S, \widehat{x})\right)$,

(e) $(\nabla F(\widehat{x}))^{*}\left(C^{+}\right)+N(S, \widehat{x})=X^{*}$. 
Proof. For the given $\widehat{x} \in S$, we take $F(\hat{x}, x)=f(x)-f(\widehat{x})$ for all $x \in X$. Then $\hat{x}$ is a local sharp efficient solution for (VOP) if and only if it is a local sharp efficient solution for (VEP). Moreover, $F_{\widehat{x}}$ is strictly differentiable at $\hat{x}$ if and only if $f$ is strictly differentiable at $\hat{x}$. When $S$ is convex, the $C$-convexity of $F_{\widehat{x}}$ on $S$ is equivalent to the $C$-convexity of $f$. Together with Theorem 7 and Corollary 12, we complete the proof.

Theorem 14. Let $\hat{x} \in S$ and let $T: X \rightarrow \mathbb{L}(X, Y)$ be $a$ mapping. Suppose that $S$ is a closed subset of $X$.

(i) If $\hat{x}$ is a local sharp efficient solution for (VVI), then one has

$$
0_{X^{*}} \in \operatorname{int}\left((T(\widehat{x}))^{*}\left(C^{+} \cap \mathbb{B}_{Y^{*}}\right)+N_{c}(S, \widehat{x})\right) .
$$

(ii) Suppose that $S$ is convex. Then the following assertions are equivalent:
(a) $\hat{x}$ is local sharp efficient solution for (VVI),
(b) $\hat{x}$ is sharp efficient solution for (VVI),
(c) $0_{X^{*}} \in \operatorname{int}\left((T(\hat{x}))^{*}\left(C^{+} \cap \mathbb{B}_{Y^{*}}\right)+N(S, \widehat{x})\right)$,
(d) $0_{X^{*}} \in \operatorname{int}\left((T(\widehat{x}))^{*}\left(C^{+}\right)+N(S, \widehat{x})\right)$,
(e) $(T(\hat{x}))^{*}\left(C^{+}\right)+N(S, \widehat{x})=X^{*}$.

Proof. Similar to the proof of Theorem 13, we take $F(\hat{x}, x)=$ $\langle T(\hat{x}), x-\widehat{x}\rangle$ for the given $\hat{x} \in S$ and for all $x \in X$. Then $\hat{x}$ is a local sharp efficient solution for (VVI) if and only if it is a local sharp efficient solution for (VEP). Moreover, since $T(\widehat{x}) \in \mathbb{L}(X, Y), F_{\widehat{x}}$ is obviously strictly differentiable at $\widehat{x}$ and $C$-convex on $S$ whenever $S$ is a convex subset of $X$. Combined with Theorem 7 and Corollary 12, this completes the proof.

\section{Acknowledgments}

The authors are grateful to the two anonymous reviewers for their valuable comments and suggestions, which helped to improve the paper. This research was partially supported by the National Natural Science Foundation of China (Grant no. 11071267).

\section{References}

[1] X. H. Gong, "Efficiency and Henig efficiency for vector equilibrium problems," Journal of Optimization Theory and Applications, vol. 108, no. 1, pp. 139-154, 2001.

[2] X.-H. Gong, "Optimality conditions for Henig and globally proper efficient solutions with ordering cone has empty interior," Journal of Mathematical Analysis and Applications, vol. 307, no. 1, pp. 12-31, 2005.

[3] X.-H. Gong, "Optimality conditions for vector equilibrium problems," Journal of Mathematical Analysis and Applications, vol. 342, no. 2, pp. 1455-1466, 2008.

[4] X.-H. Gong, "Scalarization and optimality conditions for vector equilibrium problems," Nonlinear Analysis, vol. 73, no. 11, pp. 3598-3612, 2010.

[5] Q. H. Ansari, "Vector equilibrium problems and vector variational inequalities," in Vector Variational Inequalities and Vector Equilibria, F. Giannessi, Ed., vol. 38, pp. 1-16, Kluwer Academic, Dordrecht, The Netherlands, 2000.
[6] Q. H. Ansari, I. V. Konnov, and J. C. Yao, "Existence of a solution and variational principles for vector equilibrium problems," Journal of Optimization Theory and Applications, vol. 110, no. 3, pp. 481-492, 2001.

[7] Q. H. Ansari, X. Q. Yang, and J.-C. Yao, "Existence and duality of implicit vector variational problems," Numerical Functional Analysis and Optimization, vol. 22, no. 7-8, pp. 815-829, 2001.

[8] Q. H. Ansari, I. V. Konnov, and J. C. Yao, "Characterizations of solutions for vector equilibrium problems," Journal of Optimization Theory and Applications, vol. 113, no. 3, pp. 435-447, 2002.

[9] M. Bianchi, N. Hadjisavvas, and S. Schaible, "Vector equilibrium problems with generalized monotone bifunctions," Journal of Optimization Theory and Applications, vol. 92, no. 3, pp. 527-542, 1997.

[10] N. Hadjisavvas and S. Schaible, "From scalar to vector equilibrium problems in the quasimonotone case," Journal of Optimization Theory and Applications, vol. 96, no. 2, pp. 297-309, 1998.

[11] B. Jiménez, "Strict efficiency in vector optimization," Journal of Mathematical Analysis and Applications, vol. 265, no. 2, pp. 264284, 2002.

[12] M. Studniarski, "Necessary and sufficient conditions for isolated local minima of nonsmooth functions," SIAM Journal on Control and Optimization, vol. 24, no. 5, pp. 1044-1049, 1986.

[13] R. T. Rockafellar and R. J.-B. Wets, Variational Analysis, vol. 317, Springer, Berlin, Germany, 1998.

[14] D. E. Ward, "Characterizations of strict local minima and necessary conditions for weak sharp minima," Journal of Optimization Theory and Applications, vol. 80, no. 3, pp. 551-571, 1994.

[15] X. Y. Zheng, X. M. Yang, and K. L. Teo, "Sharp minima for multiobjective optimization in Banach spaces," Set-Valued Analysis, vol. 14, no. 4, pp. 327-345, 2006.

[16] F. Flores-Bazán and B. Jiménez, "Strict efficiency in set-valued optimization," SIAM Journal on Control and Optimization, vol. 48, no. 2, pp. 881-908, 2009.

[17] S. J. Li, S. K. Zhu, and X. B. Li, "Second-order optimality conditions for strict efficiency of constrained set-valued optimization," Journal of Optimization Theory and Applications, vol. 155, no. 2, pp. 534-557, 2012.

[18] X. Q. Yang and X. Y. Zheng, "Approximate solutions and optimality conditions of vector variational inequalities in Banach spaces," Journal of Global Optimization, vol. 40, pp. 455-462, 2008.

[19] S. K. Zhu, S. J. Li, and X. W. Xue, "Strong Fermat rules for constrained set-valued optimization problems on Banach spaces," Set-Valued and Variational Analysis, vol. 20, no. 4, pp. 637-666, 2012.

[20] J.-P. Aubin and H. Frankowska, Set-Valued Analysis, vol. 2, Birkhäuser Boston, Boston, Mass, USA, 1990.

[21] J.-P. Aubin and I. Ekeland, Applied Nonlinear Analysis, John Wiley \& Sons, New York, NY, USA, 1984.

[22] F. H. Clarke, Optimization and Nonsmooth Analysis, John Wiley \& Sons, New York, NY, USA, 1983.

[23] B. S. Mordukhovich, Variational Analysis and Generalized Differentiation, Vol I: Basic Theory, Vol II: Applications, Springer, Berlin, Germany, 2006.

[24] J. Jahn, Vector Optimization, Springer, Berlin, Germany, 2004.

[25] E. Kreyszig, Introductory Functional Analysis with Applications, John Wiley \& Sons, New York, NY, USA, 1978.

[26] J. Burke and S.-P. Han, "A Gauss-Newton approach to solving generalized inequalities," Mathematics of Operations Research, vol. 11, no. 4, pp. 632-643, 1986. 


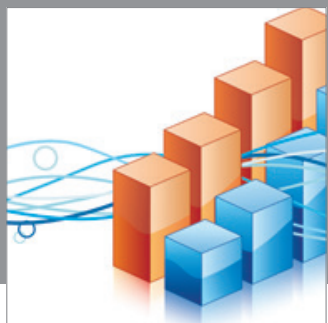

Advances in

Operations Research

mansans

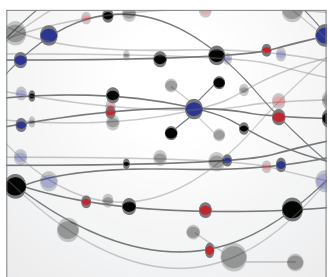

The Scientific World Journal
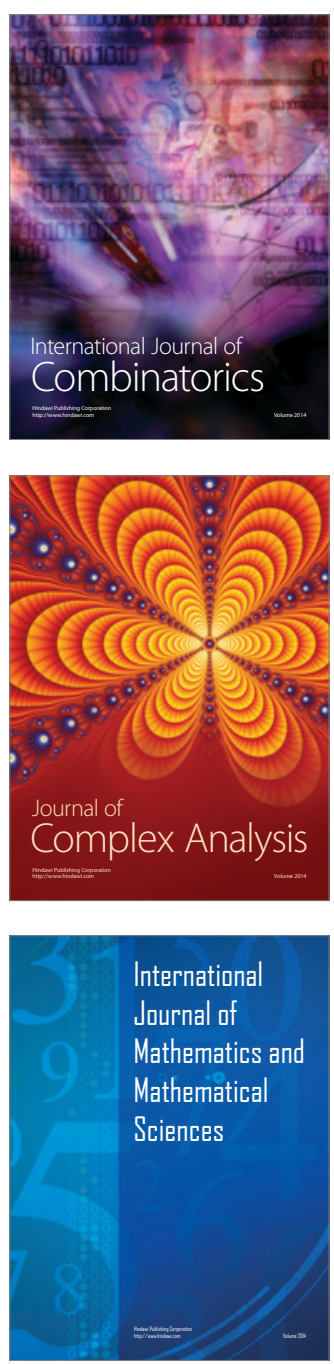
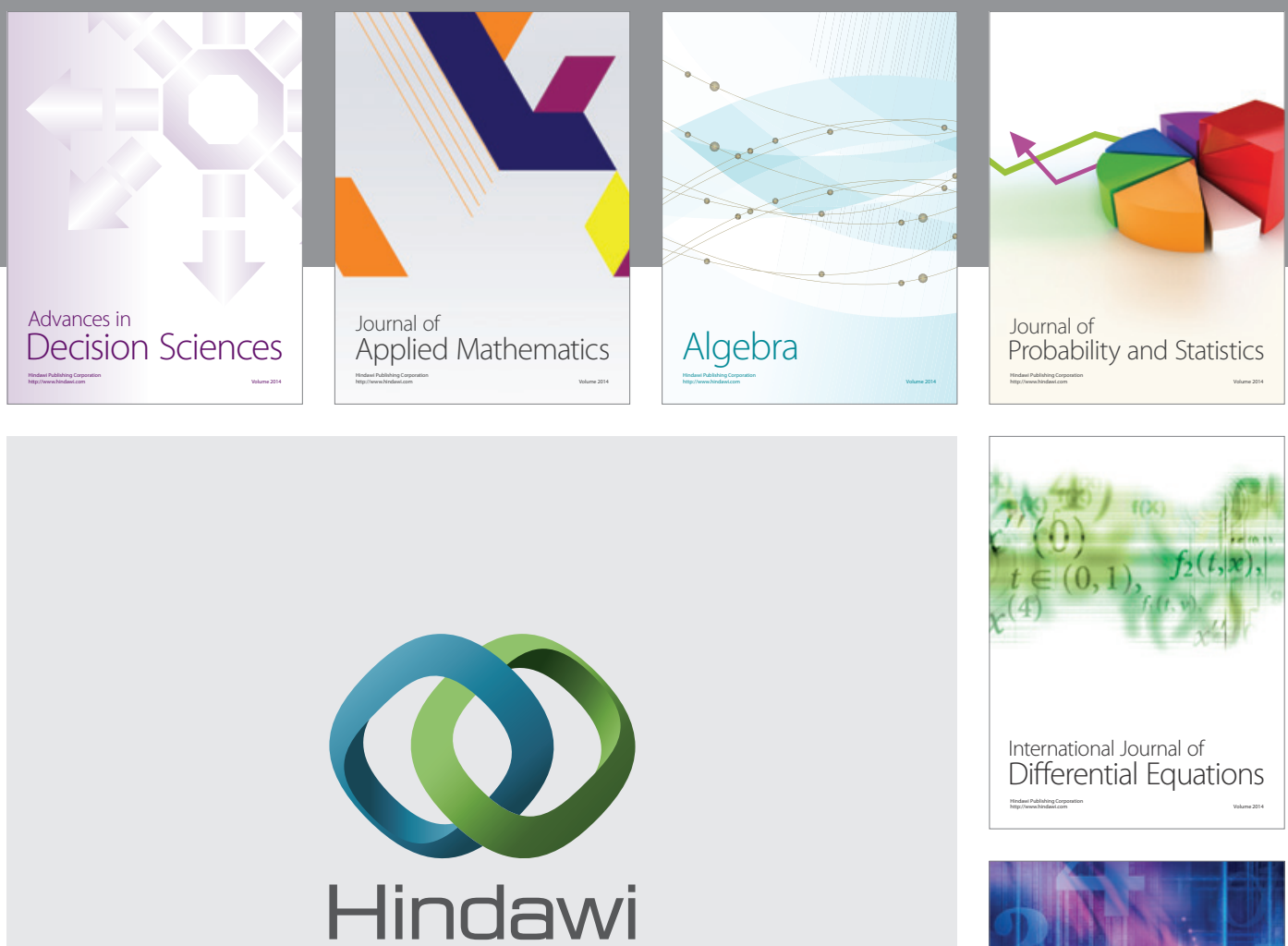

Submit your manuscripts at http://www.hindawi.com
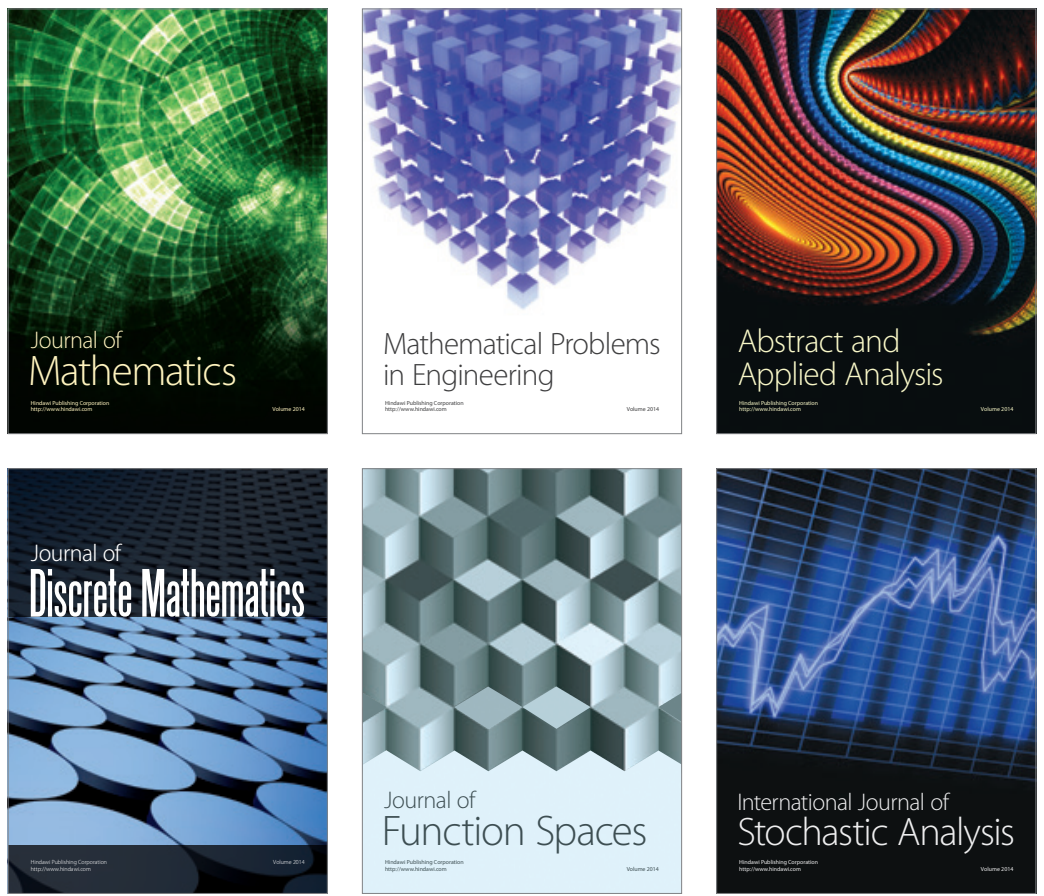

Journal of

Function Spaces

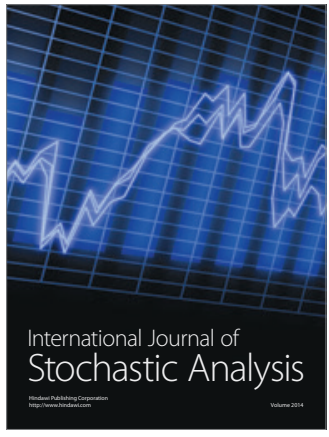

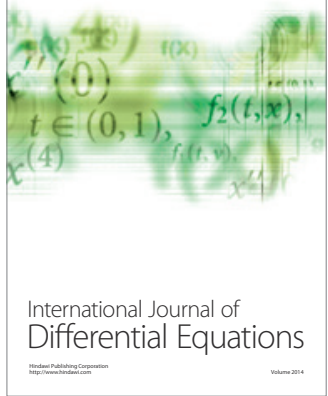
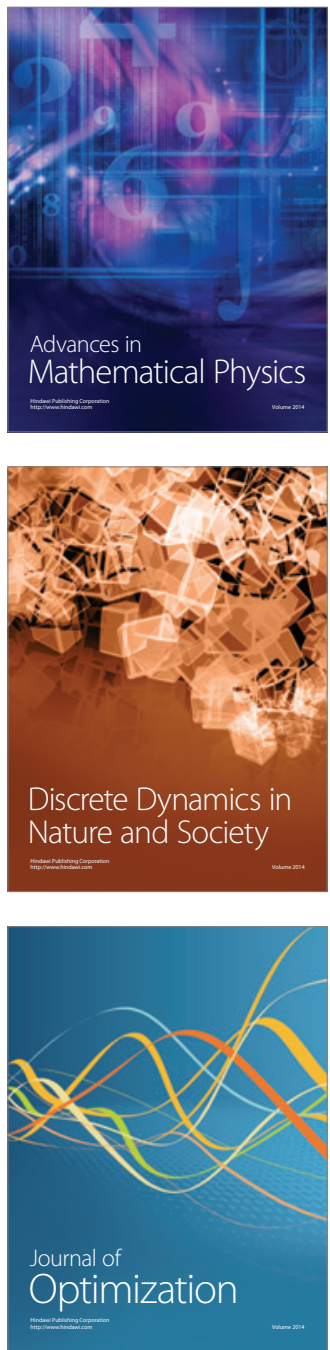